\title{
The Process of Developing a Multi-Cell KEMS Instrument
}

\author{
E. H. Copland ${ }^{\mathrm{a}}$, J. V. Auping ${ }^{\mathrm{b}}$, and N. S. Jacobson ${ }^{\mathrm{b}}$ \\ ${ }^{\text {a }}$ CSIRO CMSE, Lindfield, NSW 2070, Australia \\ Formerly with CWRU/NASA Glenn Research Center, Cleveland, OH USA \\ ${ }^{\mathrm{b}}$ Structures and Materials Division, NASA Glenn Research Center, \\ Cleveland, $\mathrm{OH} 44135$ USA
}

\begin{abstract}
Multi-cell KEMS offers many advantages over single cell instruments in regard to in-situ temperature calibration and studies on high temperature alloys and oxides of interest to NASA. The instrument at NASA Glenn is a $90^{\circ}$ magnetic sector instrument originally designed for single cell operation. The conversion of this instrument to a multi-cell instrument with restricted collimation is discussed. For restricted collimation, the 'field aperture' is in the copper plate separating the Knudsen Cell region and the ionizer and the 'source aperture' is adjacent to the ionizer box. A computer controlled $x-y$ table allows positioning of one of the three cells into the sampling region. Heating is accomplished via a Ta sheet element and temperature is measured via an automatic pyrometer from the bottom of the cells. The computer control and data system have been custom developed for this instrument and are discussed. Future improvements are also discussed.
\end{abstract}

\section{Introduction}

Knudsen Effusion Mass Spectrometry (KEMS) is a powerful tool for thermodynamic measurements and have been used at NASA Glenn Research Center for many years. Key parameters generated by this instrument are an input into long term recession predictions for high temperature materials, parameters for predictions of fiber/matrix interactions in composites at high temperatures, and understanding vapor deposition processes. Multi-cell KEMS offers many advantages over conventional single cell KEMS. The main advantage is that it provides an internal reference, which is constant and not subject to changes in machine calibration constant when samples are changed. In addition a multiple cell provides a temperature calibration point (e.g. triple point of $\mathrm{Au}$ ) and a standard material (e.g. Au, Ag) can provide a continual check of instrument operation throughout an experiment. The idea was first proposed by Buchler and Stauffer in 1966 (1) and has been applied by a number of investigators since. However to fully realize the benefits of such an approach, a number of critical factors must be considered and incorporated into the instrument. The first is restricted collimation (2), which leads to the ionizer effectively sampling only the effusate from a particular cell and also leads to a constant ionization volume. The second is accurate positioning of the particular cell being sampled. The implementation of restricted collimation and the development of multi-cell flange and furnace with accurate positioning will be discussed in this paper. 
The instrument at NASA Glenn is a Nuclide/MAAS/Patco 12-90-HT single-focusing magnetic sector instrument, purchased about 22 years ago. Over the years, the instrument has been heavily modified. A diagram of the instrument is given in Fig. 1. This instrument has been described in detail in a recent report (3).

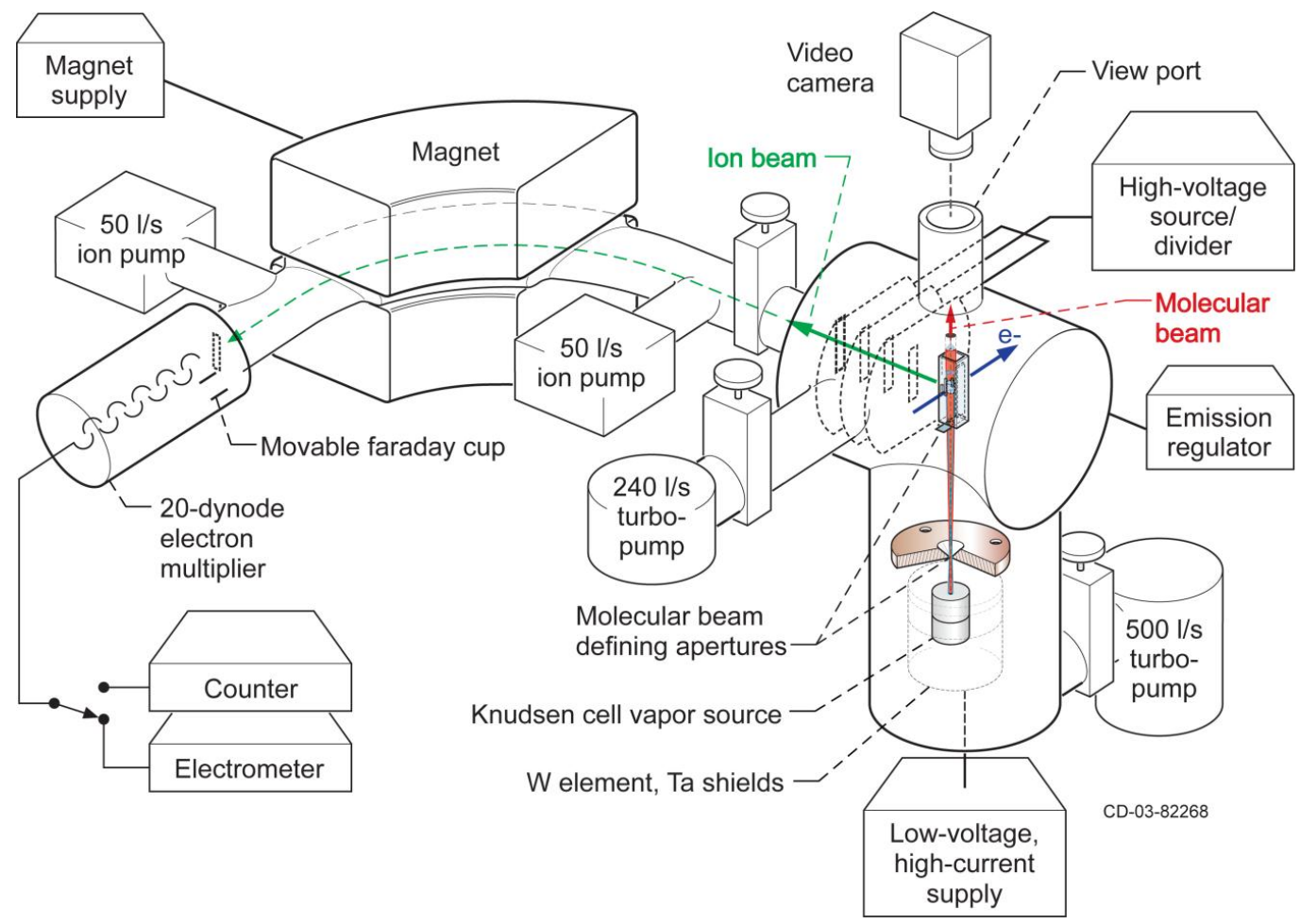

Figure 1. Diagram of KEMS instrument at NASA Glenn.

The NASA Glenn instrument has a cross-axis ionizer with the molecular beam, electron beam, and ion beam mutually perpendicular. The ion source is non-magnetic and ion detection is now done exclusively with ion counting. Ideally, this approach leads to measurements which are virtually free of mass discrimination effects. In a magnetic sector instrument the mass-tocharge ratio is proportional to the square the inverse magnetic field. With ion counting, no mass corrections are needed.

The Knudsen cell chamber and the ionization chamber are pumped by magnetically levitated turbo pumps, backed by dry-scroll pumps. The region in the magnet and the multiplier section are pumped by ion pumps. In addition the ionization chamber has a liquid nitrogen cooled cold finger. The combination of these and the high resolution of the instrument (Res 1100) help minimize the effects of any hydrocarbon background.

\section{Restricted Collimation}

Chatillon and co-workers have shown that restricted collimation is particularly beneficial in minimizing general background and improving reproducibility of sampling (2). In this case 
restricted collimation means a series of small apertures which define the molecular beam and lead to a constant ionization volume at a fixed location in the ionizer. This is illustrated with a simple 'ray diagram' in Fig. 2(a). Note that with restricted collimation, the ionizer effectively 'sees' only inside the effusion cell and spurious molecules from outside the cell being sampled (including the cells not being sampled) ideally do not reach the ionizer.

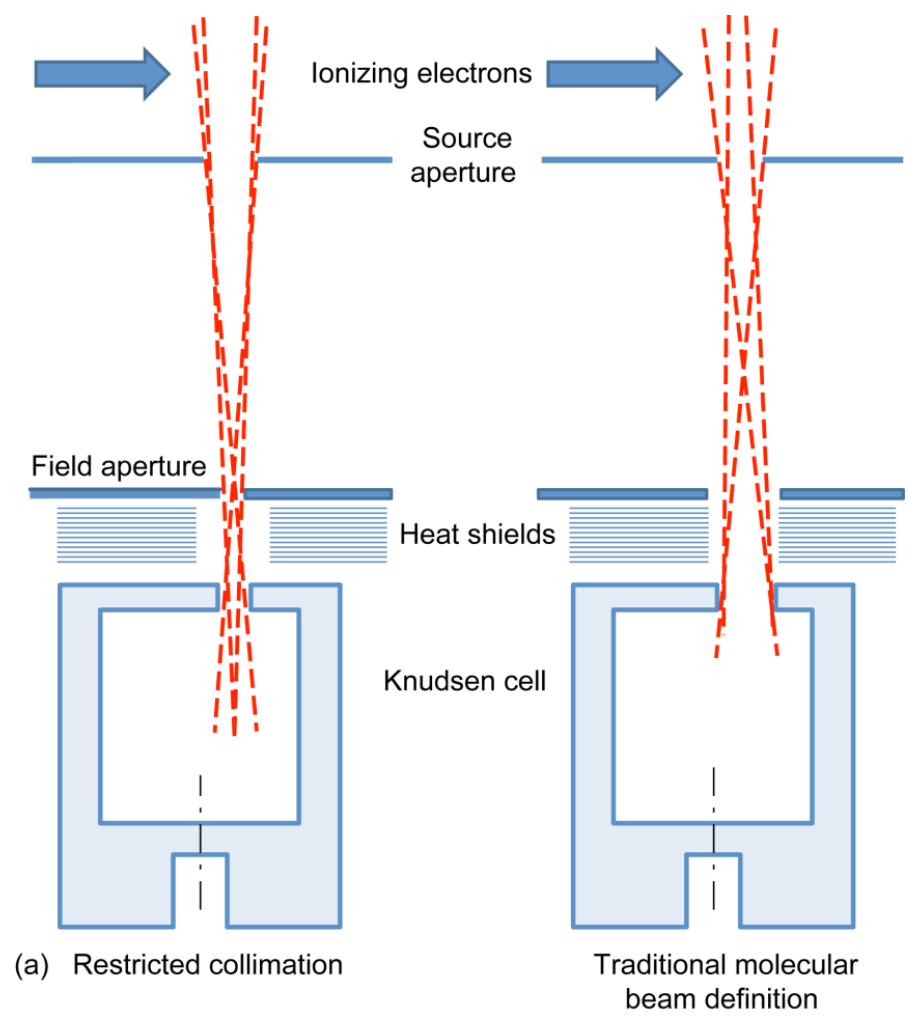

Fig. 2(a). Ray diagram, which shows the advantages of restricted collimation over traditional molecular beam definition.

Figure 2(b) illustrates the implementation of restricted collimation and multi-cell vapor source on the NASA Glenn instrument. The field aperture is part of the copper plate which separates the Knudsen cell chamber from the ionizer chamber. The source aperture is built into ionizer, as shown in Fig. 3. Not shown is a shutter, which is moveable metal plate between the field aperture and the source aperture. Chatillon and co-workers (2) have described in detail the process of optimizing the cell orifice to field aperture and field aperture to source aperture distance for maximum signal. In practice, these distances are fixed by the constraints of the instrument. The calculations of Chatillon and co-workers (2) suggest the shortest possible distance between the cell orifice and field aperture is particularly critical for maximum signal.

Most of our studies are done with channel orifices (length-to-diameter ratio of $\sim 3$ ) on the Knudsen cell. These create an ellipsoidal effusate distribution, as shown in Fig. 4. The field aperture samples at the top of the distribution. As described in the next section, the cells are translated in the $\mathrm{x}-\mathrm{y}$ plane and thus the effusate is sampled from the most intense point. 


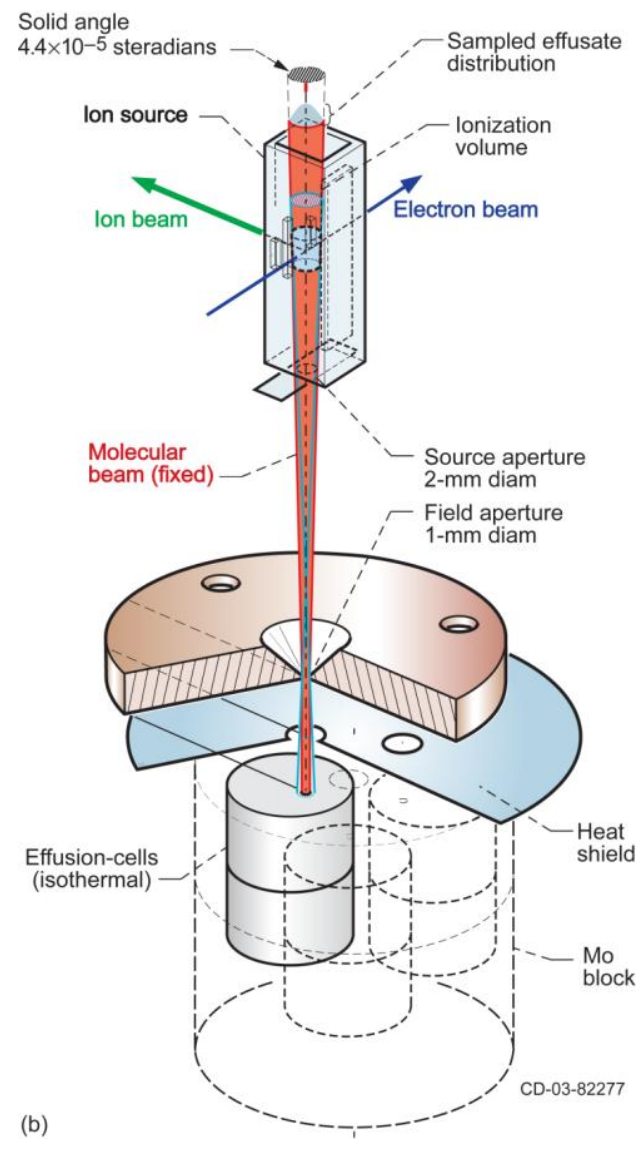

Figure 2(b). Restricted collimation on the NASA Glenn instrument.

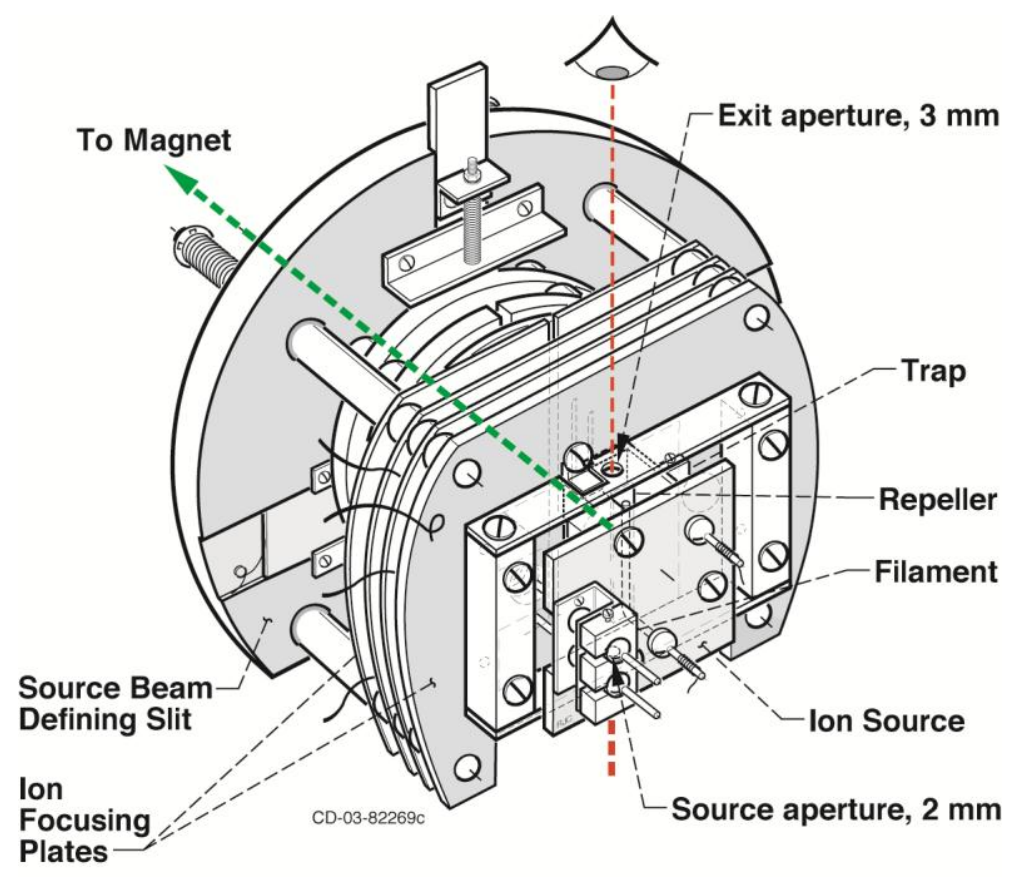

Figure 3. Ionizer, showing location of source aperture. 


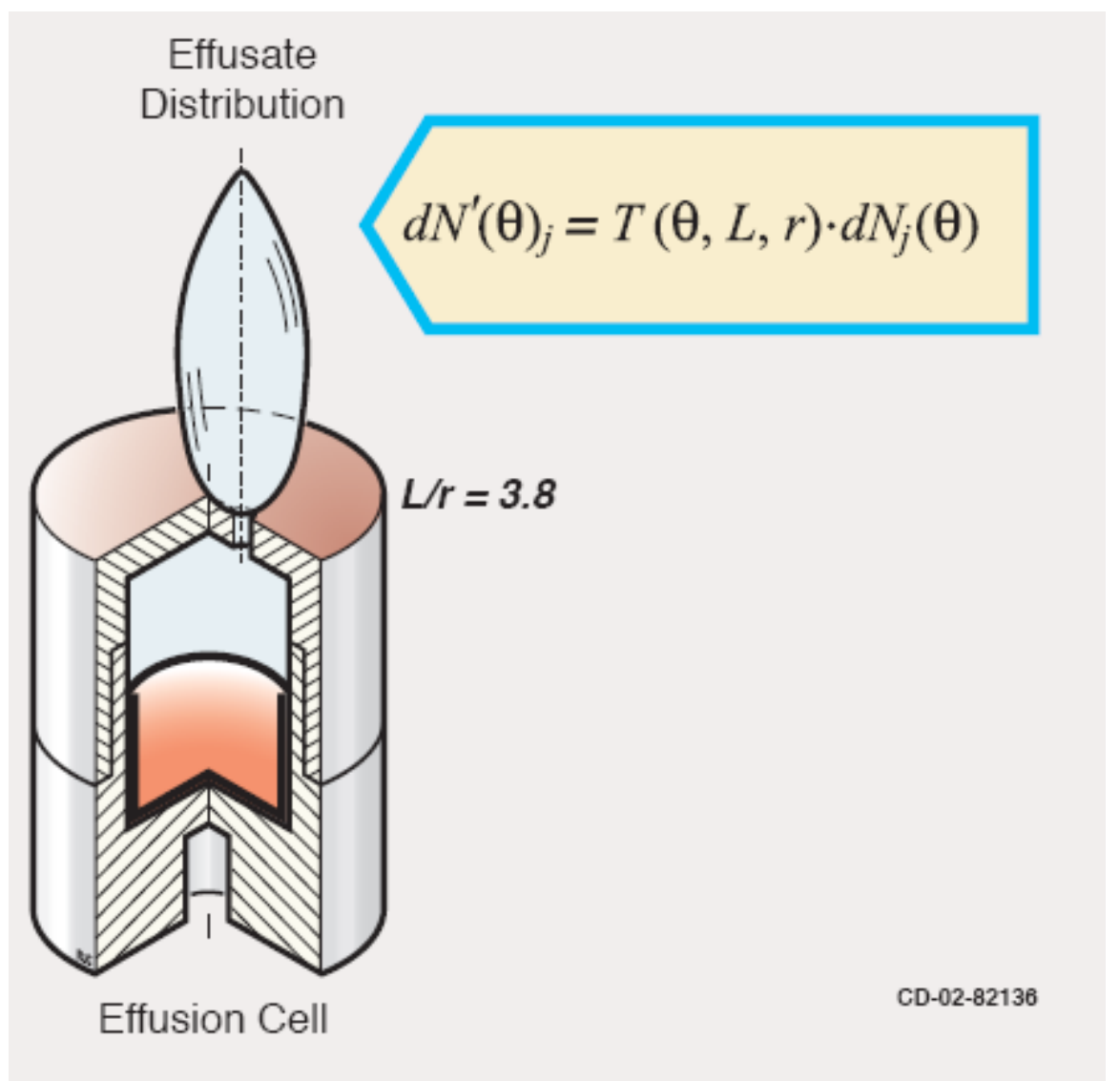

Figure 4. Effusion cell showing ellipsoid effusate distribution from

\section{Multi-Cell Flange}

The multi-cell flange is shown in Fig. 5. The three cells are translated in the $x$-y plane. The original instrument vacuum chamber $(\phi=12.5 \mathrm{~cm})$ was used for this flange, so it was critical to make effective use of this small space to attain adequate translation. The $x-y$ table was fabricated at NASA Glenn and utilized roller bearings. Stepper motors on the outside of the vacuum chamber set the position to $\pm 10 \mu \mathrm{m}$. As described in the next section, these motors are controlled by computer and a cell centering routine was developed for centering a particular cell each time it is moved into the sampling position.

The three cells are mounted in a Ta or Mo isothermal cylindrical envelope, as shown in Fig. 5. This envelope is heated with a Ta or $\mathrm{W}$ foil heating element. In the current configuration, temperatures to $2000 \mathrm{~K}$ could be attained. Temperature is measured with an automatic brightness sensing pyrometer (Micron M190-VTS) sighting into the dead-end hole at the bottom of the cell, as shown in Fig. 4. The pyrometer can be separately translated and tilted for precise alignment to sight into the center of the dead-end hole.

Space limitations only allow temperature to be measured on one cell. To confirm the validity of this and the constant temperature of the block, three cells with Au were placed in the 
block. The ion intensity ratios were measured for each and are shown in Fig. 6. As required, the values are near unity. It has been found that difference sets of cells gave different ion intensity ratios. This conferences that these ratios are due to the geometry factor, whose origins are in the small machining differences between each cell. Typically it should be as close to one as possible, as shown in the figure. This indicates the cylindrical envelope is isothermal. The geometry factor is then applied to correct for the minor difference between cells.

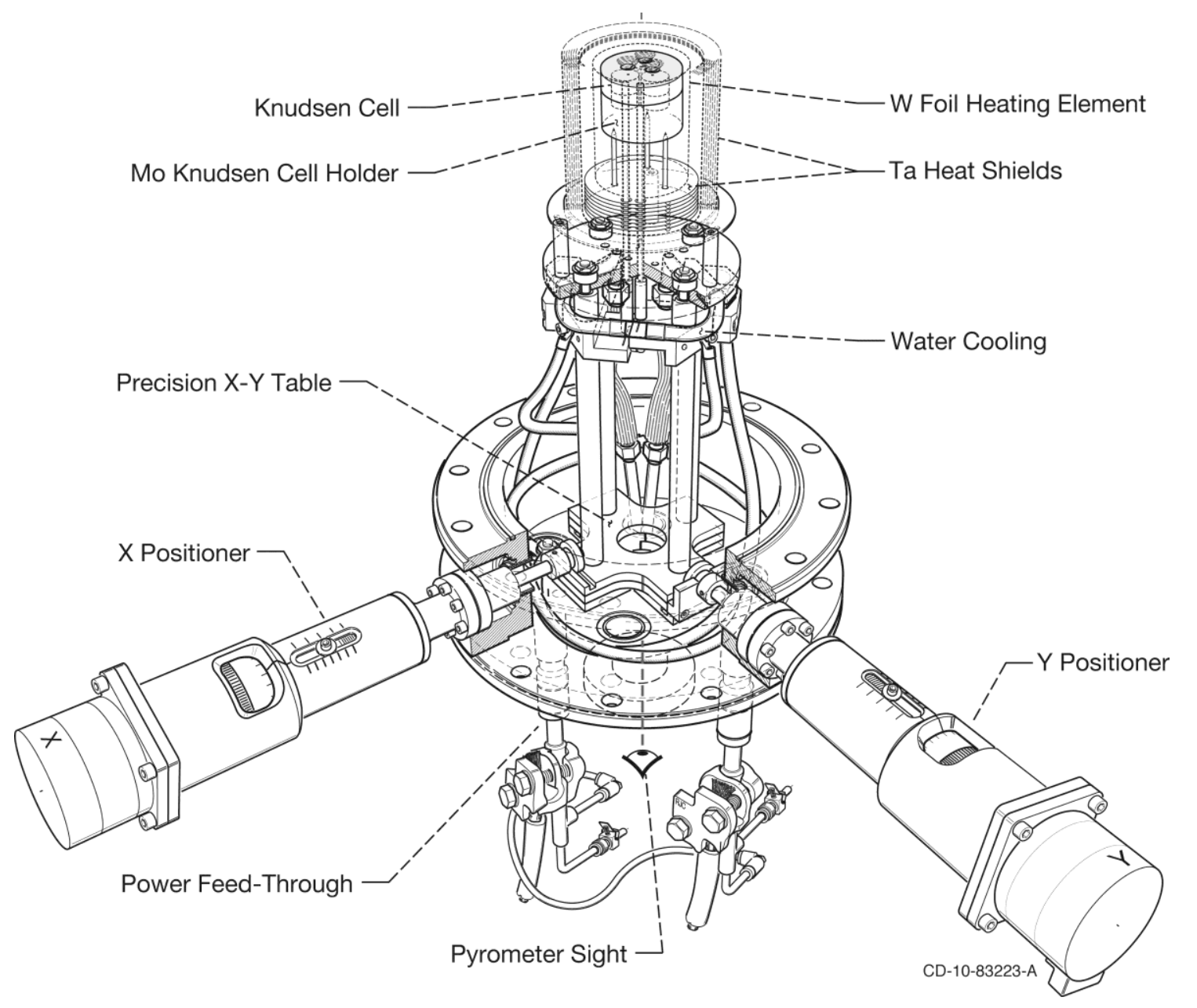

Figure 5. Schematic of multi-cell KEMS flange.

As noted, a major advantage of the multi-cell flange is the ability to perform frequent temperature calibrations - before, after, and sometimes during each experiment. This was done via the usual method of the thermal arrest in the melting point of Au. In addition, the heat of vaporization of $\mathrm{Au}$ (or other well-characterized standard) could be measured throughout every experiment, providing a continual check of proper instrument operation 


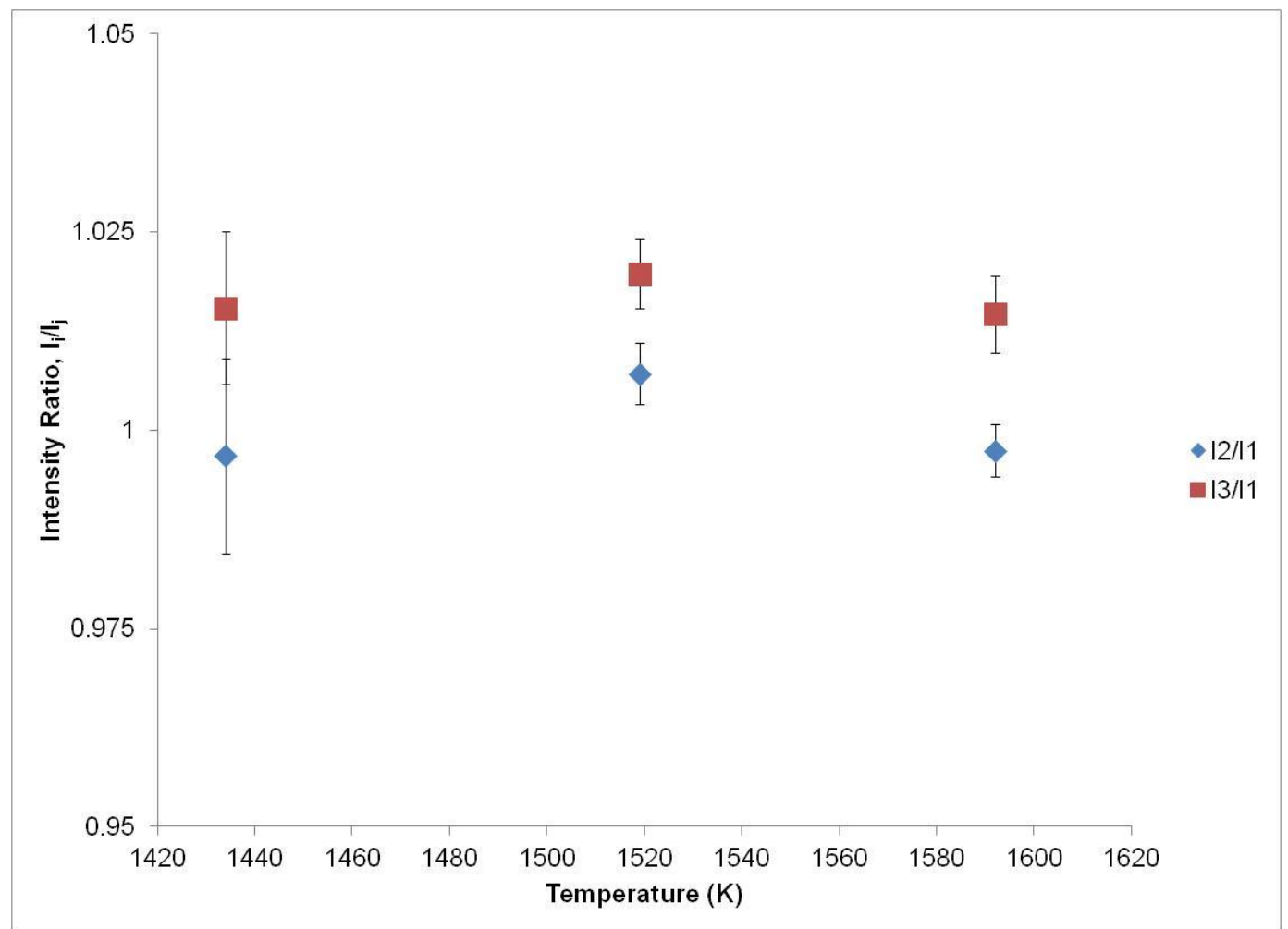

Figure 6. Measured intensity ratios for cells (2) referenced to cell (1) and cell (3) referenced to cell (1). The near unity values indicate isothermal conditions for all three cells.

\section{Computer Control and Data Acquisition}

Today most mass spectrometers have some type of computer control. In fact, many applications allow completely automated operation. We feel there so many different types of KEMS experiments and so many operator judgements to made in a particular KEMS experiment (e.g. is equilibrium attained, are there different vapor species at different temperatures, is a phase change occurring) that full automation is not feasible. However partial automation adds significantly to the versatility of the instrument.

The major functions of mass scanning, ionizing electron energy control, furnace control and temperature measurement (via either a thermocouple or a pyrometer) are controlled by the computer. In addition, the computer controls cell positioning via the stepper motors which control the $\mathrm{x}-\mathrm{y}$ table. A special window is opened for individually scanning in the $\mathrm{x}$ and $\mathrm{y}$ directions for maximum peak intensity. This is shown in Fig 7. 


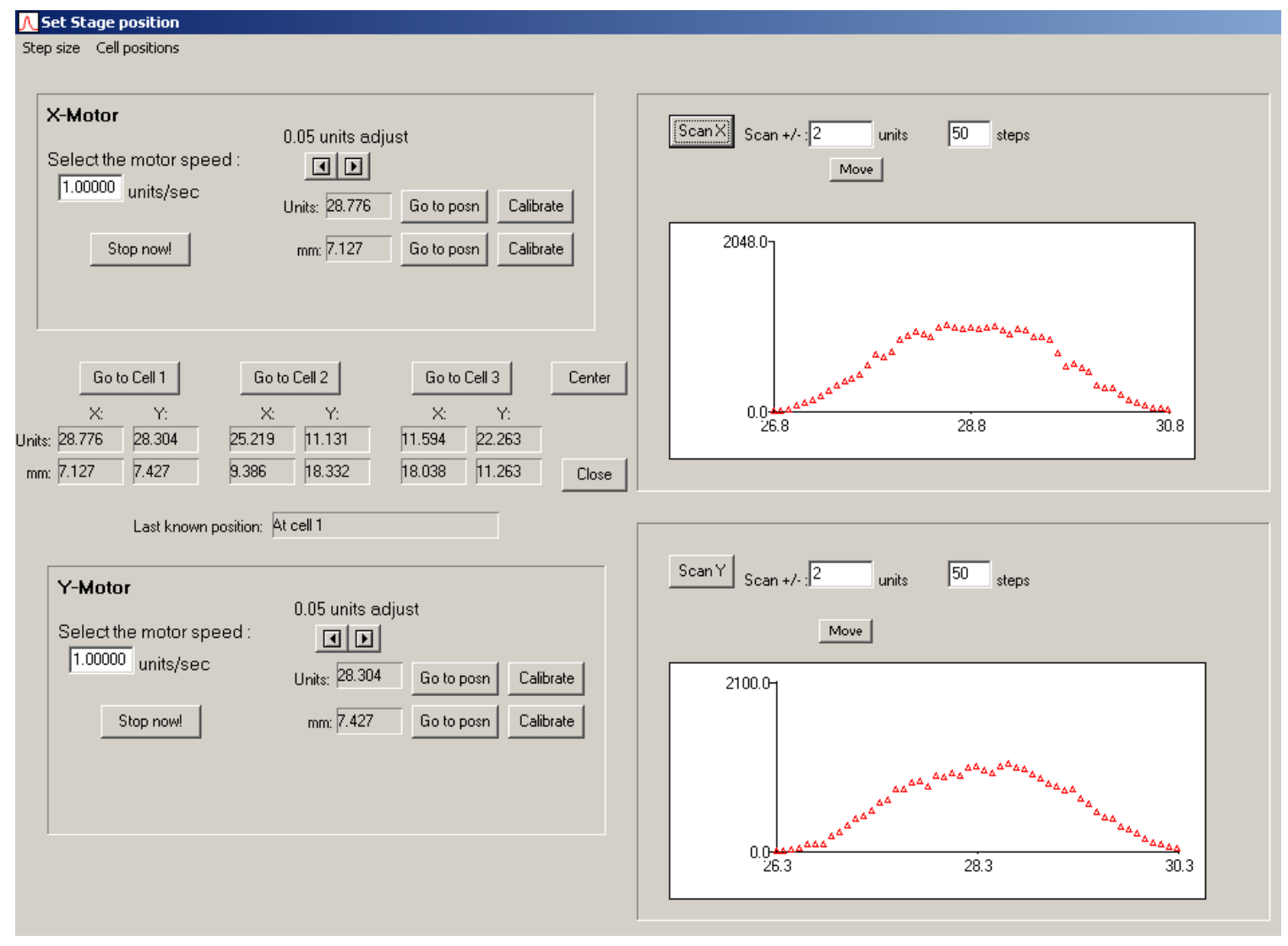

Figure 7. Positioning control for multiple cells.

Another important algorithm in our data acquisition system is the automated peak height routine, illustrated in Fig. 8. This is based on the flat top peaks attained when ion intensity is scanned vs mass number. First, the peak is scanned and the data smoothed, using 3 or 5 point averaging. Then a first derivative is taken of the scan. From the maximum and the minimum of the first derivative plot, the mid-point of the rise and mid-point of the fall are determined. These numbers are refined by using the slopes of the rise and fall to re-calculate the mid-points. Then the peak center and peak width are determined. From these, the middle $60 \%$ of the data points are averaged. The application allows repeated scanning of an individual peak to improve the signal-to-noise ratio. This routine can also be implemented during a temperature ramp. Repeated scanning allows gives peak intensity as function of temperature and allows the thermal arrest during melting to be recorded for a temperature calibration. 


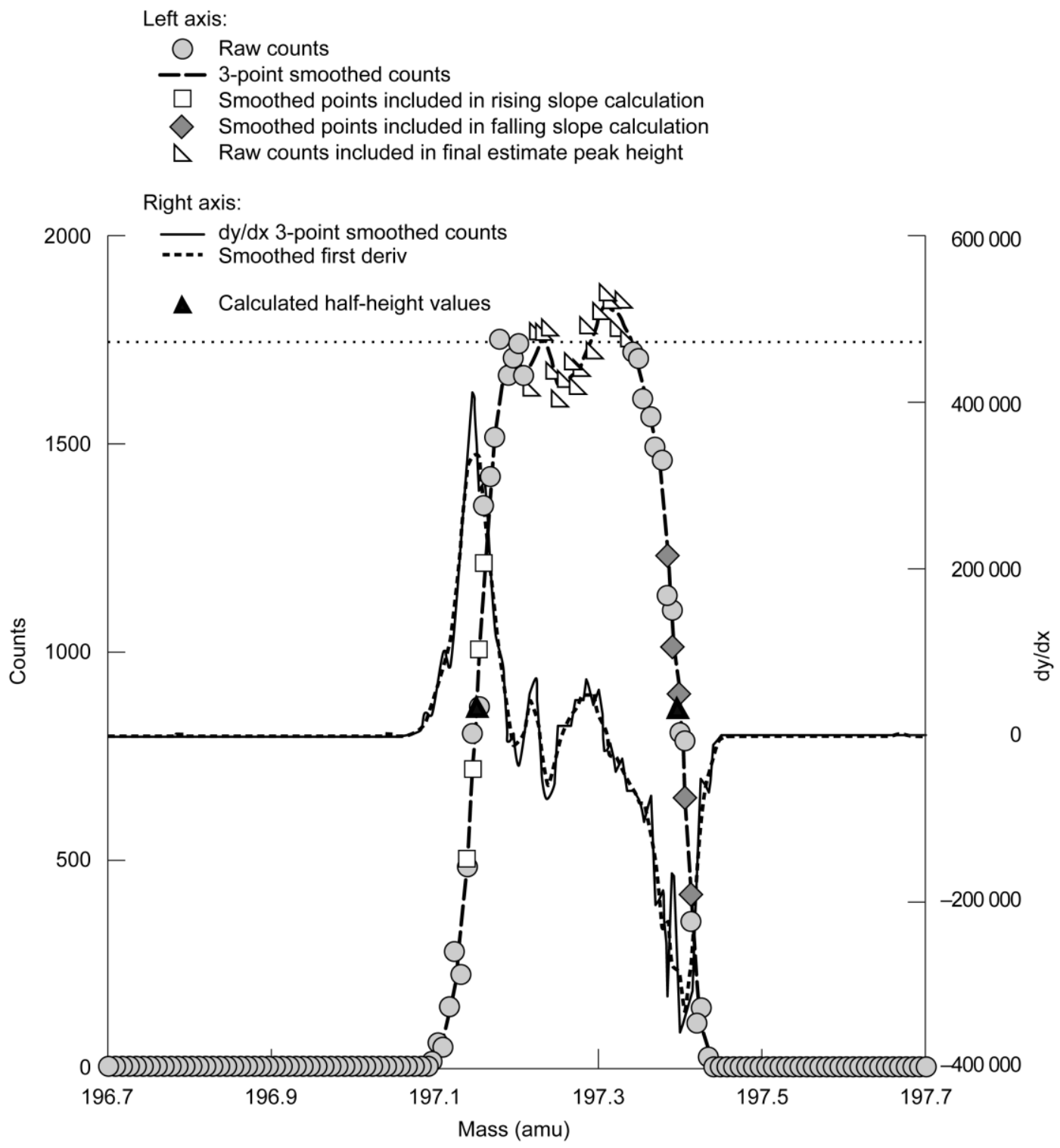

Figure 8. Illustration of peak height algorithm.

\section{Example Studies}

The NASA Glenn multi-cell system has been used for a number of alloy studies and is currently being used for oxide studies. These are based on the primary relationship in KEMS, which relates vapor pressure and ion intensity (equation [1a]). The thermodynamic activity of an alloy component, $a(i)$, is simply the ratio of the ion intensity from component $i$ in the alloy to the ion intensity of the pure material $i$ (equation [1b]):

$p(i)=\frac{k I_{i} T}{\sigma}$ 
$a(i)=\frac{p(i)}{p^{o}(i)}=\frac{I(i)}{I^{o}(i)} \frac{C_{I I}}{C_{I}^{o}}$

Here $p(i)$ is the partial pressure of component $i$, the superscript ${ }^{\circ}$ refers to the pure component, $I_{i}$ is the ion intensity of that component (assuming no fragmentation), $T$ is the absolute temperature, $C_{I I}^{o} / C_{I}$ is the geometry factor for a pair of cells described earlier, and $\sigma$ is the ionization cross section. In practice, where the activities are low $\left(\sim<10^{-3}\right)$, there is necessarily a large disparity between vapor pressure of the component in the alloy and in the pure material. In order to avoid such problems as chamber contamination from a high vapor pressure reference, a secondary reference, such as Au is used. In this case the relevant equation is:

$a(i)=\frac{p(i)}{p^{o}(A u)}\left[\frac{p^{o}(A u)}{p^{o}(i)}\right]=\frac{I(i)}{I^{o}(A u)} \frac{\sigma(A u)}{\sigma(i)} \frac{C_{I I}}{C_{I}^{o}}\left[\frac{p^{o}(A u)}{p^{o}(i)}\right]$

Fig. 8(a) and 8(b) illustrate measurement on several Ti-Al-O alloys illustrating the effect of oxygen on $\mathrm{Ti}$ and $\mathrm{Al}$ activity (4), respectively.

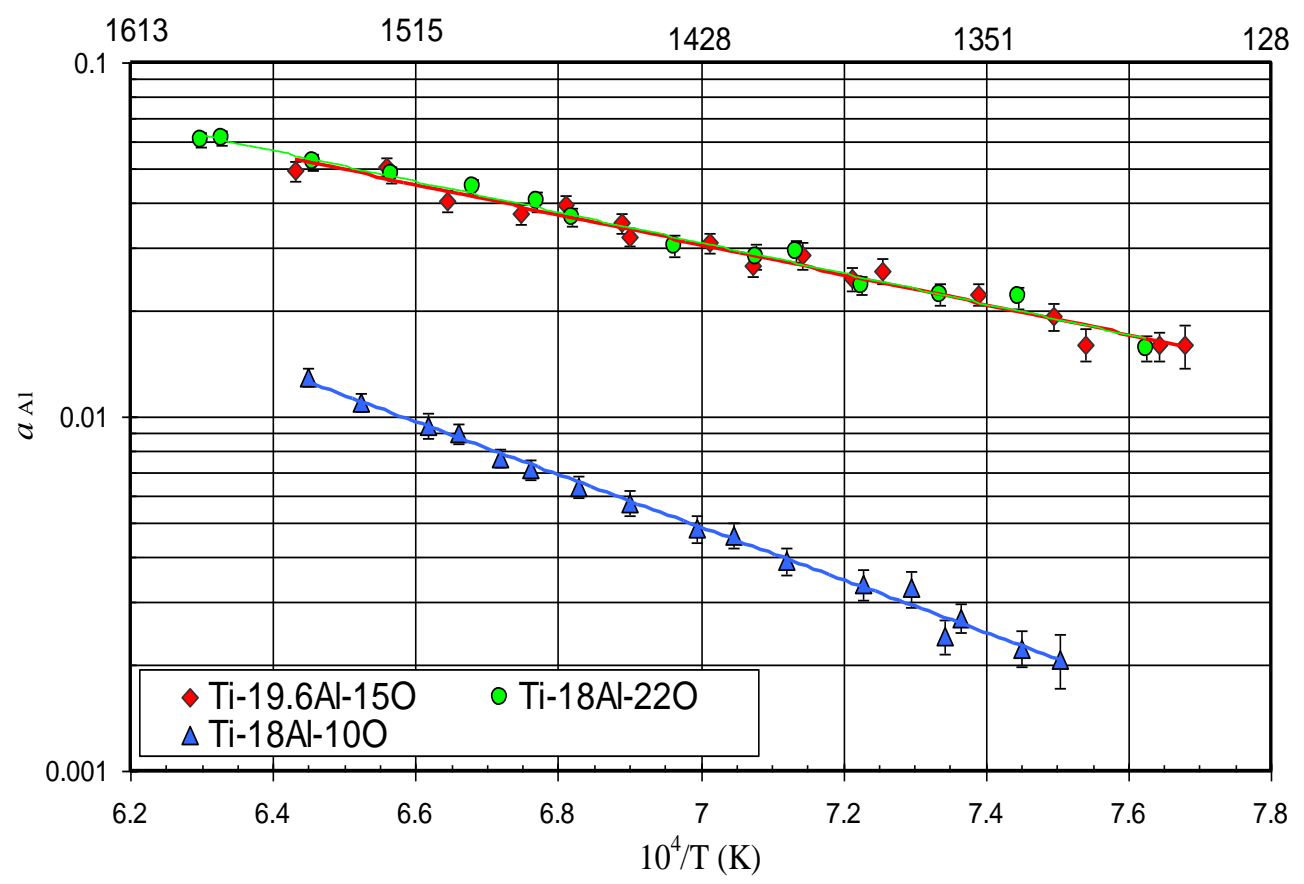

Figure 8(a). Effect of oxygen in Ti-Al-O alloys on $\mathrm{Al}$ activity. 


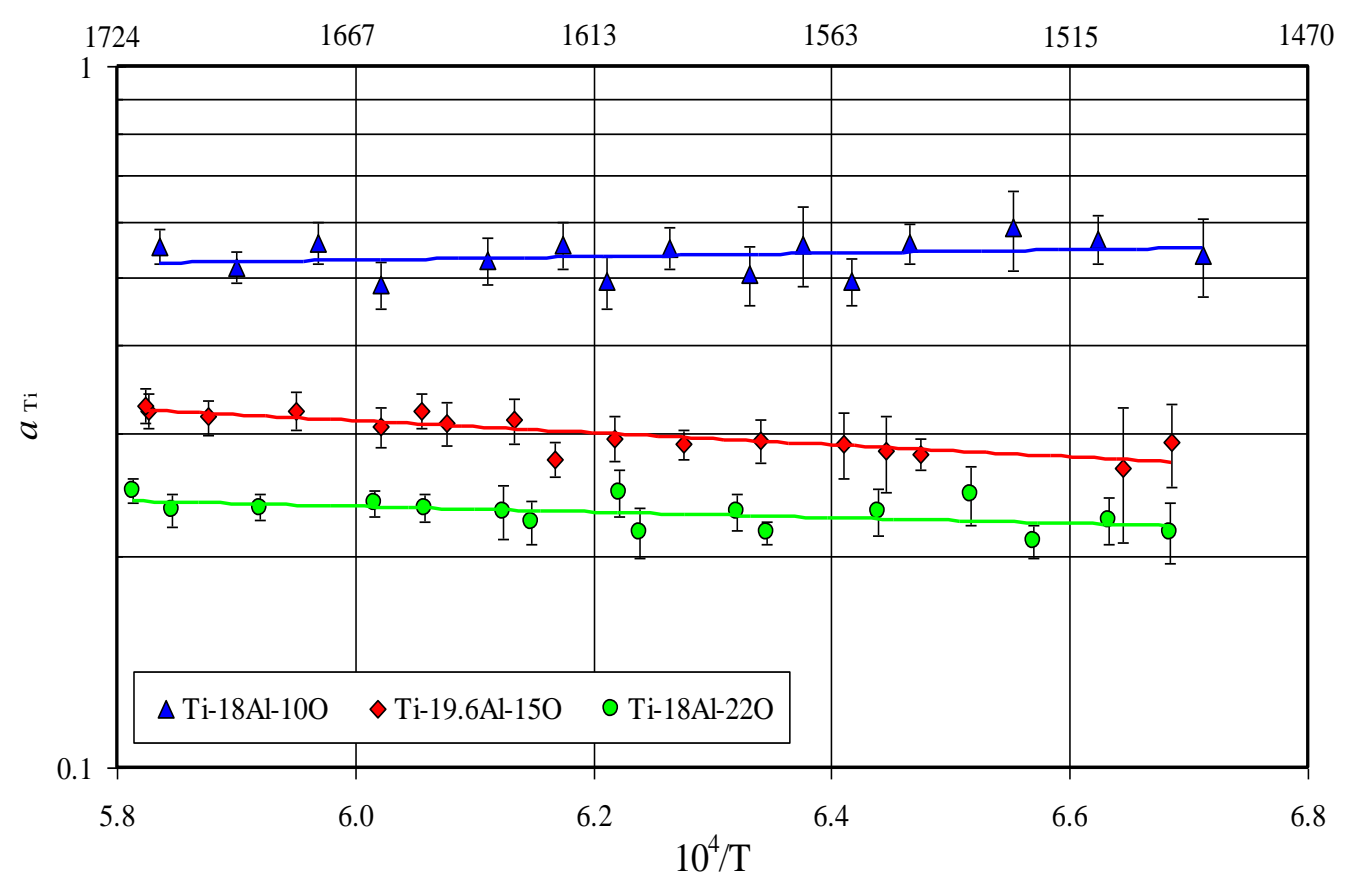

Figure 8(b). Effect of oxygen in Ti-Al-O alloys on Ti activity. Increasing oxygen concentration decreases Ti activity.

Another important application of this technique is to determine phase boundaries. Ion current ratios are very sensitive indicators of phase transformations, as proposed by Gokcen et al. (5) and later applied by Kato (6). The discontinuities in ion current ratios (e.g. the L/ $\gamma$ phase boundary is illustrated in Fig. 9(a) for the Ni-Al-O system (7). These observations led to revisions in the solidification behavior of $\mathrm{Ni}_{3} \mathrm{Al}$, as shown in Fig. 9(b), including the following:

$$
\text { Peritectoid } \beta+\gamma \rightarrow \gamma^{\prime}
$$

$$
\text { Eutectic } \mathrm{L} \rightarrow \beta+\gamma
$$

Figure 9(b) illustrates the revised phase diagram superimposed on the older phase diagram (7). Note that now there is a narrow rectangular region which allows for the two invariants above. This illustrates the value of KEMS in clarifying features of an important phase diagram. 


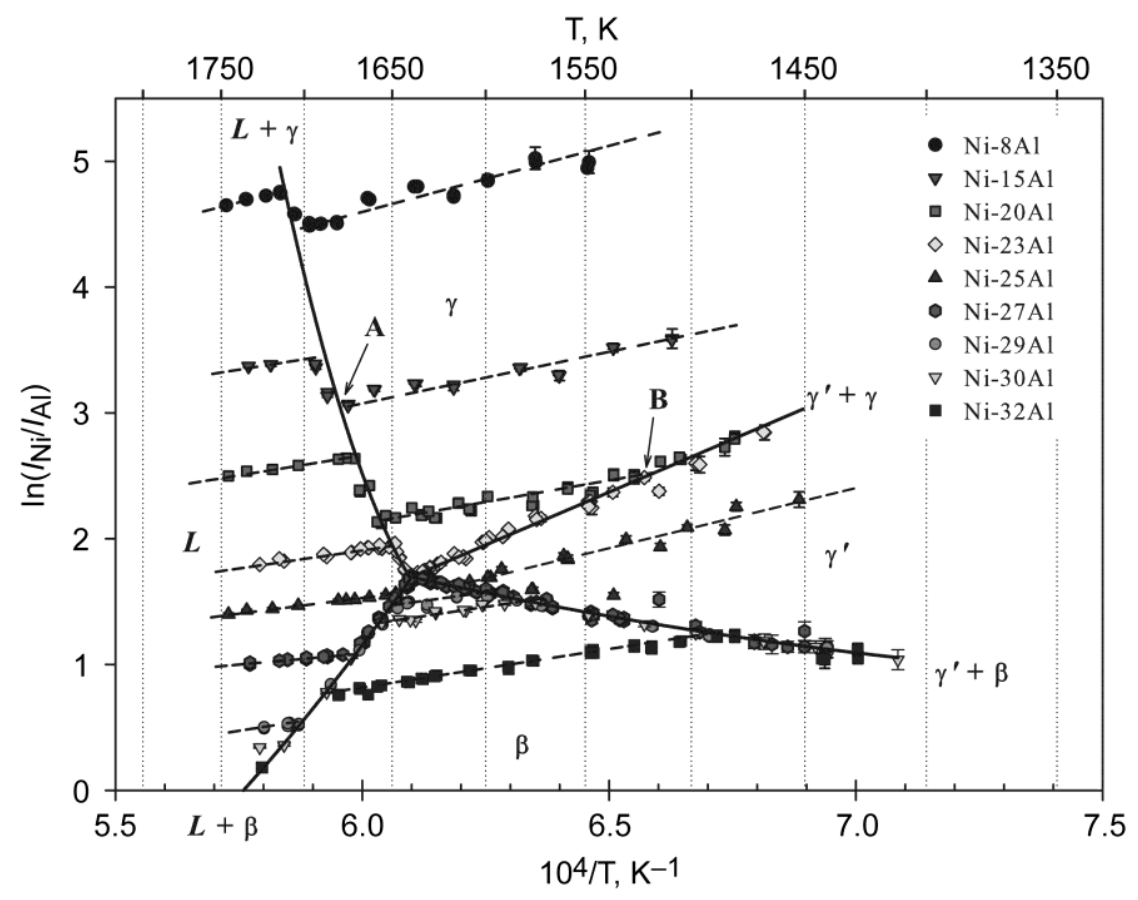

Figure 9(a)

Figure 9(a). Illustration of the use of ion current ratios to detect phase changes in the Ni-Al-O system (reprinted with permission from (7)).

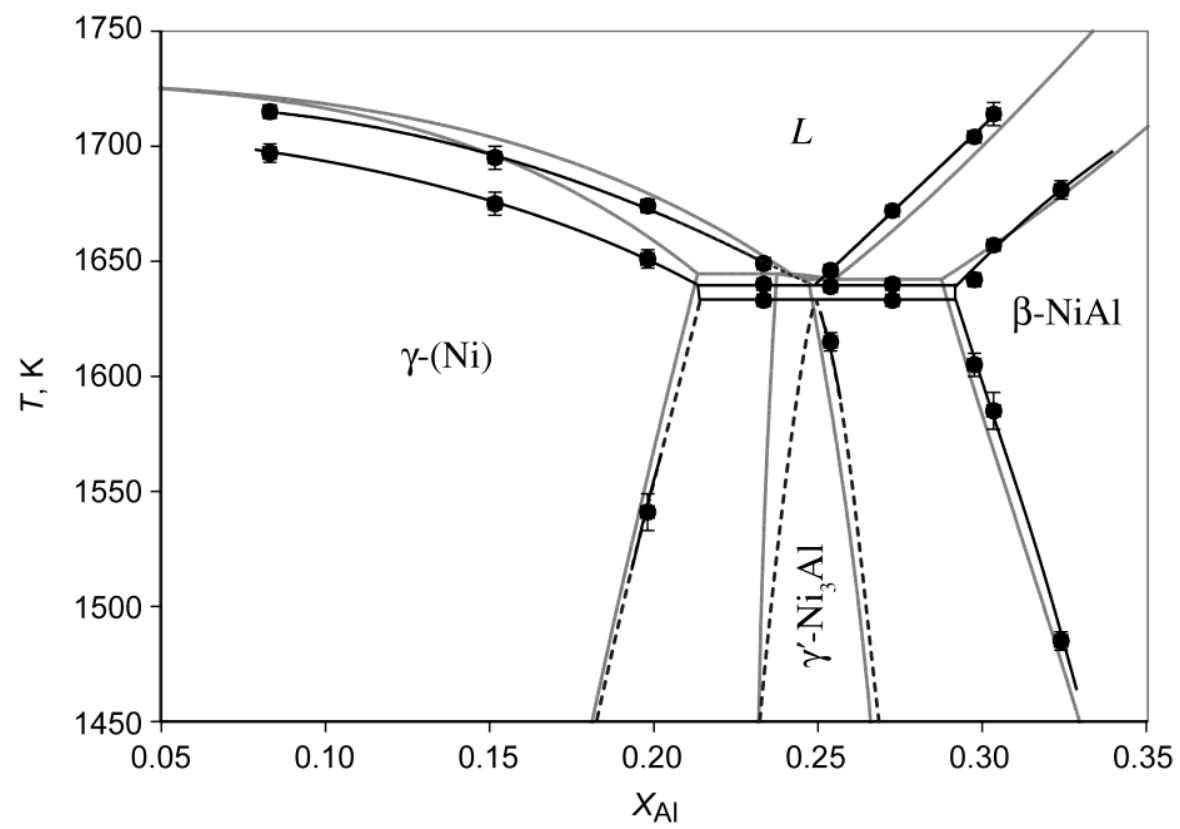

Figure $9(\mathrm{~b})$

Figure 9(b). Phase diagram for a portion of the Ni-Al system. The lines with the data points are the revised phase boundaries (7) from the KEMS study (reprinted with permission from (7)). 


\section{Future Directions}

Clearly, there are many more improvements that can be made for these type of measurements. Some type of total flux measurement would be very useful to obtain more reliable partial pressures. This could be in the form of a target collection above the ionizer with a sensitive thin film thickness measurement. Corrections would need to be made to get a total flux measurement.

Better positioning, including $\mathrm{z}$ control for the Knudsen cell orifice to field aperture distance would be a good addition. Thus, this distance could be kept constant, despite temperature variations.

Improved ionizer design should be possible, particularly now when accurate computer simulations of ionizer behavior are available (8). This should give better sensitivity and control of ionizing electron energy.

\section{Summary and Conclusions}

The development of a multi-cell KEMS instrument at NASA Glenn has been discussed. This involved the conversion of a conventional single cell magnetic sector instrument to restricted collimation. The field aperture was added to the copper plate separating the Knudsen chamber and the ionization chamber and the source aperture was added to the ionizer. Without this sampling arrangement, cross contamination of the molecular beams from each cell makes multi-cell KEMS unfeasible. The multi-cell flange was constructed with a $\mathrm{x}-\mathrm{y}$ translation table which allowed positioning of the cells to within $\pm 10 \mu \mathrm{m}$. This was necessary for accurate and reproducible sampling. It is shown that this scheme with a channel orifice allows sampling from the most intense part of the effusate distribution. The three cells are located in an isothermal envelope and heated by a Ta sheet element. Temperature is measured from the bottom with an automatic pyrometer. The computer control for the system is discussed. Standard functions such as mass scans, energy of ionizing electrons, and temperature control/measurement are accomplished via the computer. The data acquisition program also has specialized functions for cell positioning and peak height determination. These changes were necessary to obtain consistent alloy thermodynamic measurements, as illustrated with sample activity measurements in Ti-Al-O alloys and phase change measurements in Ni-Al-O alloys. Finally a few comments are given on future directions for such instruments.

\section{Acknowledgments}

We thank Prof. Christian Chatillon, Grenoble, France for the many useful papers and discussions which led to the development of this system at NASA Glenn. We also thank Ben Ebihara of NASA Glenn who did the initial design of the multi-cell flange. We are also grateful to the expert machinists at Glenn who worked on various aspects of this instrument. This work was funded by NASA's Fundamental Aeronautics Program.

\section{References}


1. A. Buchler and J.L. Stauffer, Thermodynamics Vol. I, International Atomic Energy Commision, Vienna (1966).

2. P. Morland, C. Chatillon, and P. Rocabois, H. Temp. and Mat. Sci., 37(3), 167-187 (1997).

3. E. H. Copland and N.S. Jacobson, Measuring Thermodynamic Properties of Metals and Alloys with Knudsen Effusion Mass Spectrometry, NASA/TP-2010-216795.

4. E. H. Copland and N.S. Jacobson, Thermodynamics of Ti-Al-O Alloys, unpublished work (2005).

5. N. A. Gokcen, E.T. Chang, and P.C. Marx, Novel Uses of Knudsen Cells at High Temperatures, in High Temperature Technology, p. 611-627, Butterworth \& Co., London (1969).

6. $\quad$ S. Y. Nunoue and E. Kato, Met. Trans. A, 20(5), 975-976 (1989).

7. E. H. Copland, Acta Mat., 55(14), 4853-4865 (2007).

8. D. A. Dahl, Int. J. Mass Spec., 200(1-3), 3-25 (2000). 